\title{
The Effects of Incidental Similarity with a Stranger on Mimicry Behavior
}

\author{
Nicolas Guéguen*
}

Université de Bretagne-Sud, UFR LSHS, 4, rueJeanZay, BP 92116, 56321 Lorient Cedex, France

\begin{abstract}
Aims: For social psychologists, mimicry could serve a function of "social glue", binding people together and creating harmonious relationships.

Design: In three experiments, we used the paradigm of incidental similarity in order to enhance the desire to create affiliation and rapport between a participant and a stranger, and to evaluate its effect on mimicry behavior.

Findings: Undergraduates who believed they had the same first name (Study 1), birthday (Study 2) or fingerprint similarity (Study 3) as a female-target presented on a videotape were more likely to mimic the nonverbal behavior of the target than participants who did not perceive a similarity with the target.

Conclusions: These results support the notion that mimicry will help us to create affiliation and rapport because the desire to build such relations enhances when incidental similarity exists between two strangers.
\end{abstract}

Keywords: Similarity, mimicry, social relationships.

\section{INTRODUCTION}

The Chameleon effect [1] refers to the unconscious mimicry of postures, facial expressions, mannerisms and other verbal and nonverbal behaviors. Research on mimicry is not recent in psychology literature. Giles and Powesland [2] found that people mimic accents of their counterparts. The contagious effect of laughter has been found in several studies [3, 4].

It has been found that posture and mannerism are mimicked in social interactions [5, 6]. Chartrand and Bargh [1] have shown that participants were more likely to touch their own faces when they interacted with a face touching confederate who was a stranger than when they interacted with a foot shaking confederate. In the same way, the opposite effect was found when the confederate shook her foot: then the participants were more likely to shake their own feet rather than touch their own faces. When confederates were instructed to smoke in a bar laboratory, it was observed that participants imitated the smoking behavior of the confederates [7].

People mimic their counterparts in social interactions, but research found that mimicry is also associated with higher positive evaluation of the mimicker. Chartrand and Bargh [1] engaged participants in a task with a confederate who was instructed to either mimic the mannerism of the participant or to exhibit neutral, nondescript mannerisms. Compared to those who were not mimicked, participants who were mimicked by the confederate subsequently reported a higher mean of liking the confederate and described their interaction with the confederate as being more smooth and harmonious. This result is congruent with the earlier work of Maurer and

*Address correspondence to this author at the Université de Bretagne-Sud, UFR LSHS, 4, rueJeanZay, BP 92116, 56321 Lorient Cedex, France;

Fax: 33. 2.97.67.65.37; Tel: 33.2.97.01.26.63;

E-mail: nicolas.gueguen.univ-ubs.fr
Tindall [8] who found that when a counselor mimicked the arm and leg position of a client, this mimicry enhanced the client's perception of the counselor's level of empathy more than when the counselor did not mimic the client. Interacting with an embodied artificial agent in immersive virtual reality that mimics our own behavior is sufficient to influence the rating of the agent. In a more recent experiment by Bailenson and Yee [9], a virtual agent verbally presented a persuasive argument (a message advocating a campus security policy) to a participant who interacted with the agent. In half of the cases, the virtual agent mimicked the participant's head movements at a 4 second interval while for another group of participants, the agent mimicked the prerecorded movement of another participant. After the interaction, the participant indicated his/her agreement with the message delivered by the agent and gave his/her impression about the agent. It was found that the virtual mimicking agent was more persuasive and received more positive trait ratings than the non mimicking one.

Rapport and affiliation are also associated with mimicry. LaFrance [10] has used a longitudinal design to explore the relation between a measure of nonverbal synchrony and selfreport indications of rapport with college students. She found that posture sharing between the instructor and the students was positively related to rapport. According to her, postural mimicry may be influential in establishing rapport. This link between affiliation and rapport has recently been demonstrated by Lakin and Chartrand [11]. In their experiment, participants performed a first task involving a priming procedure: they were primed with words related or not to the concept of affiliation (friend, partner...). In a second unrelated task on memory, participants watched a videotape of a female-confederate who was touching her face. During this second task, it was found that the participants who were primed with the unconscious concept of affiliation mimicked more favorably the confederate seen in the videotape than when no affiliation priming was used. Thus it seems that 
affiliation and mimicry are related. This effect was recently confirmed by two experiments made by Yabar, Johnston, Miles and Piles [12] who found that participants mimicked more favorably the nonverbal behavior of members of their in-group than members of their out-group. Again, the link between the desire for affiliation and mimicry was attested. The same effect is found in situations of closer interaction. In a recent study [13], an experiment was carried out during real sessions of speed-dating in order to test the behavioral effect of mimicry in a courtship context. Young confederate women who interacted with men during such sessions were instructed to mimic some verbal expressions and nonverbal behaviors of the men, verbal expressions only, nonverbal behaviors only or not to mimic at all. Data showed that men evaluated the dating interaction and the women-confederates more positively when they mimicked them. At the end of the dating session, men expressed greater desire to meet the women-confederates who mimicked them than the same women-confederates who did not. The same effect was found when examining buyer-seller interaction when sellers were instructed to mimic or not the customers [14]. It was found that sellers who mimicked customers received more positive evaluations.

If mimicry is associated with higher liking of the mimicker and greater affiliation, several studies have found that mimicry leads to enhance pro-social behavior toward the mimicker. Van Baaren, Holland, Steenaert and Van Knippenberg [15] found in two experiments that mimicking the verbal behavior of customers in a restaurant increased the size of the tips. In their first experiment, a waitress was instructed to mimic the verbal behavior of half of her customers by literally repeating their order. It was found that the waitress received significantly larger tips when she mimicked the patrons than when she did not. In a second experiment, it was found that compared to a baseline condition, mimicry was associated with a higher rate of customers who gave a tip and was also associated with tips of larger amounts. Spontaneous helping behavior is also affected by mimicry. Van Baaren, Holland, Kawakami and Van Knippenberg [16] mimicked the posture (position of arms, legs...) of half of the participants who took part in a task in which they had to evaluate different advertisements. The experimenter, who was seated in front of the participants, mimicked or did not mimic the participant's posture. When the task was finished, the experimenter "accidentally" dropped six pens on the floor. It was found that participants in the mimicry condition picked up the pens more often (100 $\%)$ than participants in the non mimicry condition (33\%). Behavioral mimicry can also facilitate the outcomes of negotiations. In a recent study by Maddux, Mullen and Galinsky [17], it was found that mimicry facilitated the negotiator's ability to uncover underlying compatible interests and also increased the likelihood of making a deal in a negotiation in which a prima facie solution was not possible. In the same way, it was found that in a buyer-seller interaction where buyers were instructed to mimic or not the sellers, mimicry was associated with a higher sales rate and greater compliance to the sales clerk's suggestion during the selling process [14].

The above-mentioned studies show that mimicry seems to enhance social relationships. According to Lakin, Jefferis, Cheng and Chartrand [18], the relationship between mimicry and liking or pro-social behavior would be explained by the fact that mimicry could serve to foster relationships with others. This behavior could serve a function of "social glue", binding people together and creating harmonious relationships. Some research has found that more mimicry was observed when closer relationships were created experimentally. Jefferis, van Baaren and Chartrand [19] asked a participant and a confederate in dyad to answer a set of scripted questions that were impersonal or more personal. During the session, the confederate shook her foot and the behavior of the participant was measured. It was found that in the personal questions situation, the behavioral mimicry of the participant increased compared to the situation in which impersonal questions were asked. In a second study by these authors, it was found that a recent failure of a participant to affiliate with an individual was associated with greater mimicry in a second social interaction with another confederate. Yabar, Johnston, Miles and Peace [12] found that participants mimicked more favorably an unknown female target presented in a video when the target was presented as belonging to an in-group than when she was presented as a member of an out-group. According to these authors, the results would be an empirical demonstration that mimicry is produced when affiliation and rapport between two people increase.

\section{SIMILARITY AND MIMICRY}

Regarding mimicry, incidental similarities are associated with the positive evaluation of an unknown individual who shares an incidental similarity with us. Finch and Cialdini [20] found that participants who were led to believe that they shared a birthday with Rasputin rated him less harshly. Compliance to an unknown solicitor is also associated with incidental similarities. Burger, Messian, Patel, del Prado and Anderson [21] found that undergraduates who believed they shared a birthday, first name or fingerprint similarities with a confederate were more likely to comply with a request made by the confederate. Guéguen, Pichot and Le Dreff [22] carried out an experiment in which students received an email containing a 40 question survey on their food habits which required 15-20 minutes of their time to answer. This questionnaire came from a hypothetical student of the university in which the subjects were registered. In half of the cases, the surname of the solicitor, which appeared in his/her electronic address, was the same as the surname of the target. Results showed that compliance to the request was significantly higher in the same surname condition than in the different surname condition, and that the delay in response was significantly shorter in the same surname condition than in control condition. Oates and Wilson [23] found the same effect when using a similar first name between the sender and the receiver. Such results are in keeping with Heider [24] who proposed that incidental similarities create a sense of association between people.

Based on this theory and the empirical results of previous studies on incidental similarities, Guéguen and Martin [25] made the assumption that incidental similarity between an individual and a target could influence mimicry behavior. Their study supported this assumption. In two experiments, the authors manipulated the role of incidental similarity between two individuals on mimicry behavior. Undergradua- 
tes were conducted to believe that they had ( $v s$. did not have) the same first name (Study 1) or the same subject of study (Study 2) as a target presented on videotape. The target touched her chin or scratched her cheek several times whereas the number of tactile contacts made by the participant on the chin and the cheek were counted. The authors found in both studies that participants mimicked the target behavior more frequently in the similarity condition than in the no similarity control condition.

Based on this preliminary research that connected mimicry behavior and similarity, a replication of Guéguen and Martin's (2008) study was performed in order to confirm these preliminary data (Study 1) and to test if further independent variable manipulated in order to create similarity also increase mimicry (Study 2). We expected that incidental similarity between a participant and a target would be associated with a greater level of mimicry than when no similarity or low similarity existed between a participant and a target. At least, in a third study, we manipulated the level of similarity between the target and the participant in order to test if variation in the level of similarity is associated with variation in mimicry behavior. In this third study, we expected that as soon as similarity between the target and the participant increased, participant's mimicry will increase accordingly. Three experiments were conducted in which participants were induced to believe that they shared, or did not share, a first name, a birthday or a biological characteristic with a target presented in a video-clip who expressed nonverbal behaviors. The mimicry of such nonverbal behaviors by the participant was measured and the difference was tested according to the similarity conditions.

\section{Study 1}

In study 1 , we used incidental similarity between a participant and a female-target presented in a video shown on a laptop computer by leading the participants to believe that they had the same first name as the target. The target touched her chin or scratched her cheek several times. The nonverbal behavior of the participant was filmed unobtrusively using an integrated laptop camera, and the number of tactile contacts made by the participant on the chin and the cheek were counted.

\section{Method}

\section{Participants}

Seventy-eight undergraduate women (mean age 19.3) in business and management volunteered to participate in the study. Their selection was based on the frequency of their first name in the population according to their age (source Institut National de la Statistique et des Etudes Economiques). Participants who had very usual first names (1rst quartile) or unusual first names (4th quartile) were excluded. In all, twenty-nine participants were retained to participate in the study.

\section{Materials}

Each participant in the experiment was to watch the same videotape with the sound of a 22 year old female confederate. The video, which lasted four minutes, showed a female seated in an armchair responding to a male interviewer who could be heard but was not visible in the video. The confe- derate answered a series of questions about her leisure and sport activities. While she answered the interviewer, the confederate was instructed to touch her chin or to scratch her cheek for 2 to 4 seconds. This behavior was repeated five times during the video. The video was shown on a laptop computer (a Dell XPS M1330) with a 2 mega-pixels integrated webcam.

\section{Procedure}

Participants signed up for what they believed to be a study on the way in which people form an impression about someone without directly interacting with the person. As we used a between-subject design, participants were divided into two experimental groups based on a previous random assignment. The participant was taken in charge by a male experimenter upon arriving in the experimental room, and the participant was invited to sit on a comfortable chair in front of a desk where the laptop computer was installed. No image was shown on the computer screen which was black. The experimenter explained to the participant that she had to view a videotape of a young woman who was filmed for a survey interview focused on leisure and sport activities. The experimenter added that the participant could hear the male interviewer but she could not see him because the camera which was used to make the video-clip focused only on the female-respondent. The participant was informed that she had to watch the film meticulously because she would have to respond to a series of questions about the target when the film ended. The experimenter noted that it would be preferable for the participant to have better knowledge of the target by perusing her curriculum vitae before viewing the film. He then put a folder on the desk and the participant was instructed to open it and to look at the target's CV. In both experimental conditions, the same information was given in the CV (family name, postal address, photo of the target, education, professional experience, activities, interests...). In the similarity condition, the first name of the target mentioned in the $\mathrm{CV}$ was the same as that of the participant whereas the first name was different in the non similarity condition. In the latter condition, the first name used was a usual one when the frequency of this first name in the population based on the age of the target was taken into account. The experimenter left the room for two minutes, leaving the participant alone to peruse the target's CV. Two minutes later, the experimenter returned and asked the participant if she had read the $\mathrm{CV}$ and if she was ready to view the video-clip of the target. All the participants agreed. The experimenter then pressed the space bar of the computer and the video-clip appeared on the screen. At the same time, the integrated webcam of the laptop computer began to record the participant's non-verbal behavior unobtrusively while she watched the target video-clip. The webcam was regulated to capture the bust in its entirety, the arm movements and the face of the participants. At the end of the video-clip, the word "END" appeared on the screen. The experimenter left the room while the video-clip of the target was played and returned approximately ten-twenty seconds after the end of the video-clip. The experimenter asked the participant to state what she thought the purpose of the research was and to indicate if there was anything unusual about the experiment or the material. One participant in the similarity condition was eliminated after the funnel 
debriefing because she showed some doubt about the similarity of her first name and the first name of the target. The participant was then fully debriefed and asked to give written consent for the use of the video-clip made when she viewed the video of the target (the same consent form was used in Study 2 and Study 3). Finally, the participant was thanked and left the experimental room. Two coders blind to the experimental conditions and predictions were instructed to view each video-clip of the participants separately and to count the number of times the participant touched her chin or scratched her cheek. A high inter-reliability score was found between the two coders $(r(46)=0.98, p<0.001)$.

\section{Results and Discussion}

The only dependent variable measured in this experiment was the number of tactile contacts (touch, caress, scratching the chin or the cheek) made by the participants during the four minute period in which they viewed the video-clip of the target. In the similarity condition, we found a mean of $9.43(S D=3.76)$ whereas a mean of $4.21(S D=2.81)$ was found in the non-mimicry control condition. The difference between the two conditions was statistically significant $(t(46)=5.86, p<0.001, d=1.73)$.

The results support the assumption that leading participants to believe that they had the same first name as a target enhances the mimicry of the target behavior displayed by the participants compared to a situation in which the first name of the participants and the target were different. These results confirm those found by in Guéguen and Martin's study [25]. Thus, such results confirm the robustness of the similarity manipulation on mimicry. We interpreted these results in support of the theory of Lakin, Jefferis, Cheng and Chartrand [18] that mimicry is displayed in order to create liens of affiliation and rapport. In our experiment, similarity could have increased the participant's desire for affiliation and rapport with the target given the fact that this effect was found in previous studies on helping behavior [21]. Consistent with these earlier findings, this similarity appeared to produce greater feelings of attraction toward the target, which in turn led to an increase in mimicry behavior.

\section{Study 2}

In study 2, we wanted to extend the effect of similarity on mimicry demonstrated in Study 1 and in Guéguen and Martin's (2008) study [25] by using a different incidental similarity employed in previous research which tested the effect of similarity on helping behavior $[20,25]$. In this new study, we led participants to believe that they shared a birthday with the target presented in the video. In addition, this second study had another variant that not appeared in Study 1 and in Guéguen and Martin's (2008) study [25]. Firstly, no control group was used in the previous study in which the target did not express any specific nonverbal behaviors such as touching her chin or scratching her cheek from time to time, and we had no baseline level of such behavior among our participants. Secondly, a possible bias may exist in the non similarity control condition used in Study 1 and in the Guéguen and Martin's (2008) study. Effectively, the first name used in this condition could influence the level of mimicry given the fact that the effect of first name on the perception and behavior of individuals has been found in previous studies [26]. It is possible that a decrease in the level of mimicry could be found in this control condition and that the difference in the similarity condition is not explained by similarity per se, but by a negative perception of the first name of the target in the nonsimilarity condition which in turn led to a decrease in mimicry behavior. Thus, in order to prevent such bias and to evaluate the real variation in the level of mimicry in the similarity/no similarity condition, an experiment was done with participants who were led to believe that they shared a birthday with a target who was instructed to show or not show repetitive tactile facial contact during the video-clip.

Method

\section{Participants}

One hundred and twenty-four undergraduate women (mean age 18.9) in business and management volunteered to participate in the study. In order to prevent a possible bias, a first questionnaire asking the participant some demographic information was administered. Participants who were born on important dates such as Christmas, the National Holiday (July 14 in France) were excluded. One participant was excluded from the analysis because she was born on Christmas.

\section{Materials}

Two different video-clips were used in this experiment. The video-clip used in study 1 and a new video-clip similar to the previous one, but where the target who responded to a survey about her leisure and sport activities to the same nonvisible interviewer was instructed not to touch her face during the video. Each video was shown on the same laptop computer.

\section{Procedure}

A 2 (similarity/no similarity) $\times 2$ (tactile contacts/no tactile contacts of the target's face) experimental betweensubjects design was used in this study. They were divided into four experimental groups based on a previous random assignment. The participant was taken in charge as in Study 1 and the same instructions were addressed to the participant by a male experimenter. The same Curriculum was used. However, in this study, the same first name was used in all of the experimental conditions, but precautions were taken before beginning the experiment to insure that a participant would not have a name, first name, place of birth... similar to that of the target. In the two similarity conditions, the birthday (day, month but not year) of the target was the same as the birthday of the participant, whereas in the two non similarity conditions, the birthday was completely different (day, month and year) from the birthday of the participant. After that, the experimenter proceeded as in Study 1. Two minutes was given to the participant to peruse the target's $\mathrm{CV}$ and then the participant viewed the video-clip of the target on a computer and was unobtrusively recorded as before. The experimenter asked the participant to state what she thought the purpose of the research was and to indicate if there was anything unusual about the experiment or the material. Three participants in the similarity condition were eliminated after the funnel debriefing because they showed some doubt about the similarity of their birthdays and the birthday of the target. 
As before, two coders blind to the experimental conditions and predictions were instructed to view each video-clip of the participants separately and to count the number of times the participant touched her chin or scratched her cheek. A high inter-reliability score was found between the two coders $(r(118)=0.96, p<0.001)$.

\section{Results and Discussion}

As in the previous experiment, the dependent variable measured here was the number of tactile contacts (touch, caress, scratching the chin or the cheek) made by the participants during the four-minute period in which they viewed the video-clip of the target. This measure was done for the four experimental conditions. The data are presented in Table 1.

Table 1. Mean (SD in Brackets) of the Number of Tactile Facial Contacts (Touch, Caress, Scratching the Chin or the Cheek) made by the Participants during the Four Minute Period when they Viewed the VideoClip of the Target according to the Similarity Conditions and the Presence versus Absence of such Behaviors in the Video-Clip of the Target

\begin{tabular}{|c|c|c|c|}
\hline \multirow{2}{*}{$\begin{array}{c}\text { Tactile Facial Contacts } \\
\text { of the Target }\end{array}$} & \multicolumn{3}{|c|}{ Similarity Conditions } \\
\cline { 2 - 4 } & Similarity & No Similarity & Mean \\
\hline \hline Tactile contacts & $9.06(4.01)$ & $4.40(3.09)$ & $6.73(3.55)$ \\
\hline No tactile contacts & $2.19(1.75)$ & $2.42(1.91)$ & $2.31(1.83)$ \\
\hline Mean & $5.63(2.88)$ & $3.41(2.50)$ & $4.52(2.69)$ \\
\hline $\mathrm{N}=30$ in each group
\end{tabular}

A two-way (2 (similarity/no similarity) $\times 2$ (tactile contact/no tactile contact of the target's face) ANOVA was performed with the data. A significant main effect of similarity was found $\left(F(1,116)=18.21, p<0.001, E t a^{2}=\right.$ 0.22 ) revealing that the number of times the participants touched, caressed, or scratched the chin or the cheek was higher in the similarity condition than when no similarity was manipulated. A high significant main effect of the behavior of the target was found $(F(1,116)=72.66, p$ $\left.<0.001, E t a^{2}=0.37\right)$ revealing that when the target displayed a series of tactile contacts to her chin or her cheek several times during the clip, the participant showed such tactile contacts more frequently than when these behaviors were not displayed by the target. An interaction effect between the two factors was also found in our analysis $(F(1,116)=$ 22.80, $\left.p<0.001, E t a^{2}=0.24\right)$. Complementary analysis showed that this interaction effect is explained by the two groups in which the target displayed tactile contacts on her chin or her cheek. Effectively, while the difference between the similarity/no similarity conditions was significant with participants exposed to the target who expressed such nonverbal behaviors $(t(58)=5.04, p<.001, d=1.32)$, no statistical difference was found between these two conditions in the case where the target did not display such nonverbal behaviors $(t(58)=0.49, n s, d=0.13)$. Additional analysis confirmed a significant difference between the two videoclips in the similarity condition $(t(58)=8.60, p<0.001, d=$ $2.26)$ and in the non-similarity condition $(t(58)=2.99, p$ $<0.005, d=0.79$ ).
The results provided additional support for the notion that similarity increases the mimicry behavior of the participant. In this experiment, three important results were found. Firstly, we confirmed that perceived similarity between a target and a participant led the latter to mimic the nonverbal behavior of the target more favorably, and this confirmation was demonstrated with the help of new information used to induce similarity: here, the association between the birthday of the participant and the birthday of the target. Secondly, in this experiment, it was found that participants mimicked the nonverbal behavior of the target even when no similarity was manipulated. Such results confirm previous studies $[1,11]$. Thirdly, we found in this experiment that the difference between the two similarity conditions cannot be explained by a possible methodological bias due to the information used to present the target. In our four conditions, the curriculum vitae remained the same except for the information about the birthday of the target. We believe that birthday is information which is less likely to affect the perception of the target than a first name. A methodological bias was possible in Study 1 in the non similarity condition given the fact that various social perceptions are associated with the first name even when this first name is not the same as our own [26]. To our knowledge, such various social perceptions of an individual are not affected by birthday. Thus, in our second experiment, the difference between the similarity and the no similarity conditions may only be explained by similarity.

\section{Study 3}

The results of the first two studies combined with those found in the preliminary study of Guéguen and Martin [25] have demonstrated that participants mimicked a target more favorably when they shared a similarity with the target then when they did not share one. These findings seem to show that mimicry is associated with proximity in social interacttion which is congruent with the assumption that mimicry serves to create affiliation and rapport. The pressure to create affiliation and rapport with someone who is similar to us is higher, and mimicry seems to be one of the behavioral responses to this pressure. Given this, these two studies confirm the enhancing effect of similarity on mimicry behavior. Furthermore, a third study was carried out in order to confirm this effect, to generalize the effect by using additional information to create a feeling of similarity and to test the effect of various levels of similarity on mimicry behavior. Effectively, using the correspondence of birthday or first name between a participant and a target is not free from the emotional reaction associated with such information. Previous research has found that birthday [27] or first name [26] are liable to activate positive or negative feelings. Thus, in our two previous studies, we cannot rule out that such feelings could have played a role in the participant's mimicry behavior. It would be beneficial to create similarity by using information that has no emotional significance. The second interest of this third study was to test the effect of the level of similarity on mimicry behavior. Burger, Messian, Patel, del Prado and Anderson [21] manipulated the strength of similarity by telling participants that the similarity they shared with a requester was either fairly unusual or quite common. In their experiment, the experimenter told the participants that their fingerprints were similar to those of a 
confederate. Furthermore, in one condition, the experimenter said that the type of fingerprints were rare $(2 \%$ of the population) or quite common ( $80 \%$ of the population). It was found that compliance to a confederate's request was higher in the similarity condition than in the non-similarity condition but only when the participants believed that the similarity was very uncommon. In this experiment, we used roughly the same methodology to vary the level of perceived similarity between a participant and a target and to evaluate the difference on the mimicry behavior of the participant.

\section{Method}

\section{Participants}

Eighty undergraduate women (mean age 18.7) in social sciences volunteered to participate in the study. In order to prevent a possible future bias, a first questionnaire asking the participant some demographic information was administered.

\section{Materials}

The same video-clip made for the first experiment and used in the tactile facial contracts condition in the second experiment was used in the third experiment. The video-clip was shown on the same laptop computer as in Study 1 and Study 2.

\section{Procedure}

Participants were divided into four experimental groups based on a previous random assignment. As in the previous studies, the participant was taken in charge by a male experimenter upon arriving in the experimental room. She was invited to sit on a comfortable chair in front of a desk where the laptop computer was installed. The experimenter added that he was interested by how people form impressions about someone based on some biological characteristics without directly interacting with the person. He explained to the participant that recent research has discovered that personality differences often have a biological source. The experimenter described a false study concerning blood type groups which confirmed the association between personality test scores and biological measures. The experimenter then mentioned that, in his experiment, he was interested in the effect of some other biological sources, not only on personality, but on the way that people perceived others based on a biological characteristic. He explained that, in his experiment, he was not interested in blood type groups but in fingerprint types. He added that it had been found that five types of fingerprints exist worldwide and that some types are common or very common while some others are rare or very rare. The experimenter then told the participant that he had to determine her fingerprint type. An ink pad and a blank sheet of paper were used to take the participant's thumbprint. The experimenter then gave the participant a towelette and asked her to wait one minute while he determined her fingerprint type with the help of specialized software. The experiment went to a corner of the room where a computer and a scanner were located. The experimenter then put the sheet of paper with the participant's thumbprint in the scanner and began to scan the paper. He waited one minute, pretending to use the software on his computer. Then he turned toward the subject and explained that he had determined her fingerprint type. Depending on the experimental condition, he added that her fingerprints were type $\mathrm{C}$ which was very rare $(1$ individual out of 200: $0.5 \%$ ), relatively rare $(8 \%)$, or very common $(63 \%)$. After saying that, the rest of the procedure strictly followed the procedure used in the two previous studies.

When the experimenter had viewed the video-clip, she was asked for possible suspicion. No participant expressed suspicion about the fingerprints. Again, two coders were instructed to view each video-clip of the participants separately and to count the number of times the participant touched her chin or scratched her cheek. A high interreliability score was found between the two coders $(r(78)=$ 0.99, $p<0.001)$.

\section{Results and Discussion}

As in the two previous studies, the dependent variable measured in this experiment was the number of tactile contacts (touch, caress, scratching the chin or the cheek) made by the participants during the four minute period in which they viewed the video-clip of the target. This measure was done for the four experimental conditions and the data are presented in Table 2 .

Table 2. Mean (SD in Brackets) of the Number of Tactile Facial Contacts (Touch, Caress, Scratching the Chin or the Cheek) made by the Participants during the Four Minute Period when they Viewed the VideoClip of the Target According to the Experimental Conditions

\begin{tabular}{|c|c|c|c|}
\hline $\begin{array}{c}\text { Control } \\
\text { Condition }\end{array}$ & \multicolumn{3}{|c|}{ Fingerprint Similarity Conditions } \\
\hline $\begin{array}{c}\text { No Fingerprint } \\
\text { Type Similarity }\end{array}$ & $\begin{array}{c}\text { Very Rare } \\
(\mathbf{1} / \mathbf{2 0 0})\end{array}$ & $\begin{array}{c}\text { Relatively } \\
\text { Rare (8 \%) }\end{array}$ & $\begin{array}{c}\text { Very Common } \\
(\mathbf{6 3} \%)\end{array}$ \\
\hline \hline $4.95(2.79) \mathrm{b}$ & $10.05(4.61) \mathrm{a}$ & $8.85(4.26) \mathrm{ac}$ & $6.40(3.73) \mathrm{bc}$ \\
\hline
\end{tabular}

$\mathrm{N}=20$ in each group

NOTE: Values not sharing subscripts differ significantly ( $p<0.05$, Tukey's HSD test).

A one-way ANOVA between the four groups was performed with the data and revealed a significant general difference $\left(F(3,76)=6.99 p<0.001, E t a^{2}=0.21\right)$. As shown in Table 2, a Post-Hoc test for multiple comparisons was performed (Tukey's HSD test) which showed that the very rare similarity condition was significantly different from the common similarity condition and the no similarity control condition ( $p=0.04$ and 0.008 respectively, Tukey's HSD test) while the relatively rare similarity condition was significantly different from the no similarity control condition ( $p=0.02$, Tukey's HSD test).

The findings thus replicate the effect demonstrated in the first two studies with new information used to induce similarity between the participant and the target. In this study, we found that the effect of similarity was obtained with the help of information that had no emotional significance. The participants who believed they shared a rare fingerprint type with a target mimicked more favorably the tactile behavior of the target than the participant who did not share a fingerprint. Furthermore, when the participants believed that the fingerprints they shared with the target were very common, no difference in their mimicry behavior was found compared to the situation in which no similarity was manipulated. It seems then that when the probability of having a characteristic similar to another target is high, the 
feeling of similarity is low. In turn, the pressure to create affiliation and rapport with the target was most probably not sufficient enough for the participant to express behavior that would indicate to the target that the participant needed to have affiliation and rapport with the target. Because mimicry is a behavior used to express such feelings and desire, it was congruent to show that a lower feeling of similarity was associated with a lower level of mimicry behavior, whereas a higher feeling of similarity was associated with a higher level of mimicry. In all likelihood, the manipulation of the probability of possessing the same biological characteristic as the target is sufficient to vary the level of such feelings which in turn varies the level of mimicry behavior.

\section{GENERAL DISCUSSION}

We found consistent findings in three studies that show that incidental similarity between a participant and a target increases the level of mimicry displayed by the participant. The results are in keeping with the theoretical explanation that mimicry is used to create affiliation and rapport between two individuals $[11,18,19]$. The data confirmed and extend the preliminary results found by Guéguen and Martin [25]. These authors also found that similarity increased mimicry in an experiment where similarity was manipulated with the first-name as in our study 1 . The incidental similarity used in these three experiments and in the experiments carried out by Guéguen and Martin [25] probably resulted in a perceived unit relationship and a fleeting sense of liking between the target and the participant which in turn led to enhance the level of mimicry by the participant. Participants most likely reacted as if they had interacted with a friend or someone who was familiar. Yabar, Johnston, Miles and Piles [12] recently found that female participants mimicked the nonverbal behavior of members of their in-group more favorably than members of their out-group. In their experiment, female participants viewed videotapes of two female-targets in which one was presented as a member of an in-group and the other as a member of an out-group: the participants were not Christians and the confederates in the video were presented as either a Christian (out-group member) or a non Christian (in-group member). The confederate in the video described a picture to the participant and during this period she touched/ rubbed her face. The degree of mimicry of the participant was measured during this period in relation to a baseline period. It was found that compared to this baseline period, participants touched/rubbed their face more frequently when viewing the target of their in-group whereas the frequency of this behavior decreased when the target was a member of the participant's out-group. According to the authors, the link between mimicry and the desire for affiliation could explain these results. With the member of the in-group, the desire for affiliation would be high and therefore mimicry would increase in order to inform the counterpart that the participant had a desire to maintain the relation. With the member of the out-group, the decrease in mimicry would be used in order to inform the confederate that the participant did not want to interact with her and with members of her group in the future.

Closer relationships enhance mimicry [19] and incidental similarity between two strangers certainly has the property to create a feeling of closeness that in turn leads to enhance mimicry. Mere similarity and mimicry both have the power to enhance social interaction. In our three experiments, it was found that similarity enhanced the level of mimicry expressed by an individual toward a target. It was also found that a closer similarity was necessary to affect mimicry. Sharing a personal characteristic such as a birthday or a first name or an uncommon physical characteristic seems necessary to affect mimicry behavior toward the target. If the similarity has no emotional significance as is the case for birthday [27] or first name [26], or if the characteristic shared with an individual describes a great many people, the impact on mimicry seems to be non significant. Again such results are in keeping with the theory that mimicry is expressed in order to show to the person mimicked that the mimicker wants to create a close relationship with him/her. When the level of similarity is not sufficient enough to create this desire for a close relationship, it is then not necessary for the mimicker to express a high level of mimicry. Thus, it is not necessary to display mimicry when the desire to create affiliation and rapport is not activated.

The results also added to our knowledge about the role of perceived similarity in social interactions. In three experiments using roughly the same information to create a feeling of similarity, Burger, Messian, Patel, del Prado and Anderson [21] found that incidental similarity between a participant and a requester was associated with greater compliance to the solicitor's request. Consistent with our findings, Burger et al., [21] also found that the effect of similarity on compliance was only observed when the incidental similarity shared by the participants with someone was uncommon. The same results were found by Oates and Wilson [23] who investigated whether having names in common promotes altruistic behavior in a computer mediated communication by sending e-mail requests to people on the Web. They found that sharing a first name with someone is associated with greater helping behavior (obtaining some information about the city's sports team mascot) then when no similarity existed. Furthermore, they also found that sharing a surname was associated with a greater level of helping behavior than sharing a first name, and that sharing both was associated with a higher level of help. According to these authors, the desire to be kind to the solicitor increased as the level of similarity increased, which explains why these different levels of helping behavior were found. These results support the assumption that to create close relationships and to produce behavior related to such closeness - and mimicry could be viewed as a form of behavioral expression of this feeling of closeness- a high level of similarity is necessary, and it seems that all shared characteristics do not have the same weight: for example, an uncommon physical characteristic has more weight than a very common characteristic.

Our three studies do have some limitations. If similarity enhances the mimicry behavior of the participant, the question can be raised if a further effect of mimicry will also be observed when some similarity exists between a mimicker and an individual. Research has found that an individual who mimics our own nonverbal behavior is perceived more favorably $[1,9,8]$ and is helped more frequently $[15,16]$. It would then be interesting to evaluate in future studies the effect of similarity in combination with mimicry on the evaluation of the mimicker and on helping behavior. It is 
possible that similarity associated with mimicry could create an interaction effect on judgment and helping behavior that would be higher than the mere enhancing effect of each variable.

Another question which was not addressed in our studies concerns gender effects. Effectively, in order to control possible cross-gender effects and given the characteristics of the student population in the university where the experiments were performed, we used only female participants who viewed a female-target. Furthermore, if the generalizetion of our findings across sex is necessary, we have no reason to think that the findings observed in our studies would be different with male participants. Previous research on mimicry did not find gender difference when observing mimicry of male or female participants [1].

In conclusion, in our three studies, we found that similarity between a participant and a target enhances mimicry by the participant, and the level of mimicry is related to the level of manipulated similarity. These effects support the notion that mimicry has a social function destined to favor affiliation and rapport between individuals. The effect of mimicry is astonishing for social psychologists and further studies are necessary to explain why and in which social situations this effect occurs.

\section{CONFLICT OF INTEREST}

None declared.

\section{ACKNOWLEDGEMENT}

None declared.

\section{REFERENCES}

[1] Chartrand TL, Bargh JA. The chameleon effect: the perceptionbehavior link and social interaction. J Person Soc Psychol 1999; 76: 893-910.

[2] Giles H, Powesland PF. Speech Style and Social Evaluation. London: Academic Press 1975.

[3] Bush LK, Barr CL, McHugo GJ, Lanzetta JT. The effects of facial control and facial mimicry on subjective reaction to comedy routines. Motiv Emot 1989; 12: 120-38.

[4] Provine RR. Faces as releasers of contagious yawning: An approach to face detection using normal human subjects. Bull Psychon Soc 1992; 27: 211-4.

[5] Bernieri FJ. Coordinated movement and rapport in teacher-student interactions. J Nonverbal Behav 1988; 12: 120-38.
[6] LaFrance M. Posture mirroring and rapport. In: Davis M, Ed. Interaction Rhythms: Periodicity in Commutative Behavior (pp. 279-298). New York: Human Sciences Press 1982.

[7] Harakeh Z, Engels R, van Baaren RB, Scholte RH. Imitation of cigarette smoking: An experimental study of smoking in a naturalistic setting. Drug Alcohol Depend 2007; 86: 199-206.

[8] Maurer RE, Tindall JH. Effects of postural congruence on client's perception of counselor empathy. J Couns Psychol 1983; 30: 15863.

[9] Bailenson JN, Yee N. Digital chameleons: Automatic assimilation of nonverbal gestures in immersive virtual environments. Psychol Sci 2005; 16: 814-9.

[10] LaFrance M. Nonverbal synchrony and rapport: Analysis by the cross-lag panel technique. Soc Psychol Q 1979; 42: 66-70.

[11] Lakin JL, Chartrand, TL. Using nonconscious behavioral mimicry to create affiliation and rapport. Psychol Sci 2003; 14: 334-9.

[12] Yabar Y, Johnston L, Miles L, Peace V. Implicit behavioral mimicry: investigating the impact of group membership. J Nonverbal Behav 2006; 30: 97-113.

[13] Guéguen N. 100 petites expériences en psychologie de la séduction. 2007; Paris, Dunod.

[14] Jacob C, Guéguen N, Martin A, Boulbry G. Retail salespeople's mimicry of customers: effects on consumer behavior. J Retail Consum Serv 2011; 18: 381-8.

[15] Van Baaren RB, Holland RW, Steenaert B, Van Knippenberg A. Mimicry for money: behavioral consequences of imitation. J Exp Soc Psychol 2003; 39: 393-8.

[16] Van Baaren RB, Holland RW, Kawakami K, Knippenberg A. Mimicry and prosocial behaviour. Psychol Sci 2004; 14: 71-4.

[17] Maddux WW, Mullen E, Galinsky AD. Chameleons bake bigger pies and take bigger pieces: strategic behavioral mimicry facilitates negotiation outcomes. J Exp Soc Psychol 2008; 44: 461-8.

[18] Lakin JL, Jefferis VE, Cheng CM, Chartrand T. The chameleon effect as social glue: evidence for the evolutionary significance of nonconscious mimicry. J Nonverbal Behav 2003; 27: 145-62.

[19] Jefferis VE, van Baaren R, Chartrand TL. The functional purpose of mimicry for creating interpersonal closeness. Working Paper: The Ohio State University 2003.

[20] Finch JF, Cialdini RB. Another indirect tactic of (self-) image management: Boosting. Person Soc Psychol Bull 1989; 15: 222-32.

[21] Burger JM, Messian N, Patel S, del Prado A, Anderson C. What coincidence! The effects of incidental similarity on compliance. Pers Soc Psychol Bull 2004; 30: 35-43.

[22] Guéguen N, Pichot N, Le Dreff G. Similarity and helping behavior on the Web: the impact of the convergence of surnames between a solicitor and a solicitee in a request made by e-mail. J Appl Soc Psychol 2005; 35: 423-9.

[23] Oates K, Wilson M. Nominal kinship cues facilitate altruism. Proc R Soc Lond 2002; 269: 105-9.

[24] Heider F. Psychol Interperson Relat. John Wiley: New York 1958.

[25] Guéguen N, Martin A. Incidental similarity facilitates behavioural mimicry. Soc Psychol 2009; 40: 88-92.

[26] Joubert CE. Personal names as a psychological variable. Psychol Rep 1993; 23: 1123-45.

[27] Helson C. Petite psychologie de l'anniversaire. Dunod: Paris 2007.

Received: December 25, 2011

Revised: February 20, 2012

Accepted: February 25, 2012

(C) Weiss et al.; Licensee Bentham Open.

This is an open access article licensed under the terms of the Creative Commons Attribution Non-Commercial License (http://creativecommons.org/licenses/by$\mathrm{nc} / 3.0 /$ ), which permits unrestricted, non-commercial use, distribution and reproduction in any medium, provided the work is properly cited. 